Charles University, 1st Faculty of Medicine, Department of Ophthalmology, Prague, Czech Republic E Hrdlicková-Cela M Filipec

1st Faculty of Medicine, Institute of Anatomy

E Hrdlicková-Cela

J Plzák

K Smetana Jr

1st Faculty of Medicine, Department of Otorhinolaryngology, Head and Neck Surgery

J Plzák

Centre for Cell Therapy and Tissue Repair

K Smetana Jr

1st Faculty of Medicine, Department of Pathological Physiology

Z Mělková

Ludwig-MaximiliansUniversity, Faculty of Veterinary Medicine, Institute of

Physiological Chemistry, Munich, Germany

$\mathrm{H}$ Kaltner

H-J Gabius

La Jolla Institute for Allergy and Immunology, San Diego, CA, USA F-T Liu

Correspondence to: Karel Smetana, Charles University, 1st Faculty of Medicine, Institute of Anatomy, U nemocnice 3, 12800 Prague 2, Czech Republic

ksmet@1f1.cuni.cz

Accepted for publication 16 May 2001

\title{
Detection of galectin-3 in tear fluid at disease states and immunohistochemical and lectin histochemical analysis in human corneal and conjunctival epithelium
}

\begin{abstract}
Enkela Hrdličková-Cela, Jan Plzák, Karel Smetana Jr, Zora Mělková, Herbert Kaltner,
\end{abstract} Martin Filipec, Fu-Tong Liu, Hans-Joachim Gabius

\begin{abstract}
Backgroundlaim-Components of the tear fluid contribute to the biochemical defence system of the eye. To reveal whether the immune mediator and lipopolysaccharide binding galectin-3 is present in tears, tear samples were collected from eyes in healthy and pathological states. Investigation of expression of galectin-3 and galectin-3 reactive glycoligands in normal human conjunctival and corneal epithelia was also initiated as a step to understand the role of galectin-3 in ocular surface pathology.

Methods-Immunoblot analysis using either a rabbit polyclonal or a mouse monoclonal antibody against galectin-3 was employed to detect galectin-3 in tear fluid. Galectin-3 expression in tissue specimens was detected by immunocytochemistry employing A1D6 mouse monoclonal antibody, and galectin-3 reactive glycoligands were visualised by lectin histochemistry using labelled galectin-3.
\end{abstract}

Results-Galectin-3 was found only in tears from patients with ocular surface disorders. It was expressed in normal corneal and conjunctival epithelia but not in lacrimal glands. Inflammatory leucocytes and goblet cells found in galectin-3 containing tear fluid also expressed galectin-3. Galectin-3 binding sites were detected on the surface of conjunctival and corneal epithelial cells co-localising with desmoglein.

Conclusions-This study revealed expression of galectin-3 in tear fluid obtained from patients with eye diseases. The role of this endogenous lectin (produced by inflammatory as well as epithelial cells) in antimicrobial action and inflammation modulation could be expected.

(Brf Ophthalmol 2001;85:1336-1340)

Progress in glycosciences has documented that biological information transfer not only exploits protein-protein and nucleic acid-protein interactions but also protein-carbohydrate recognition. ${ }^{1}$ Proteins involved in interaction with carbohydrates are known as lectins. Based on structural analysis of the carbohydrate recognition domains, animal lectins are currently classified into five categories: C type, I type, $\mathrm{P}$ type, galectins, and pentraxins. ${ }^{2}$ Cells as well as extracellular matrix molecules of normal and pathological corneas and conjunctivas in mammals are already known to contain glycans recognised by numerous plant lectins. ${ }^{34}$ The sugar receptors in these tissues have been demonstrated by employing labelled neoglycoligands. ${ }^{5}$ This experimental basis encourages us to further investigate expression of endogenous lectins on the eye surface. In this report, we focus on a member of the animal lectin family of the galectins. Mammalian galectins at present comprise nine proteins sharing the property of secretion via a nonclassic pathway and cation independent binding capacity to $\beta$-galactosides, including histoblood A and B group saccharides and poly-Nacetyl-lactosamines. ${ }^{25}$ Functionally, galectins have been proposed to have crucial biological roles by recognising carbohydrate ligands on intracellular and extracellular compartments and glycoproteins of the extracellular matrix, thus contributing to cell-cell and cell-matrix interaction, ${ }^{6-8}$ regulation of cell growth, ${ }^{9}{ }^{10}$ and programmed cell death. ${ }^{11}$ In the immune system they modulate different steps of the inflammatory cascade, ${ }^{12} 13$ The only chimeratype galectin, galectin-3, deserves special attention in this context.

Galectin-3 is a protein of $M_{r}$ of $29000-$ 35000 depending on the animal species, which is expressed and secreted by various types of cells, especially monocytes, macrophages, mast cells, and epithelial cells including corneal epithelium. ${ }^{2614}{ }^{15}$ It is a mitogen capable of stimulating fibroblast cell proliferation in a paracrine fashion through interaction with cell surface glycoconjugates. ${ }^{10}$ Also, this protein can exert an anti-apoptotic activity underscoring its strong effect on cell growth. ${ }^{16}$

The cornea is a transparent, avascular tissue that is exposed to the external environment. The anterior corneal surface is covered by the 


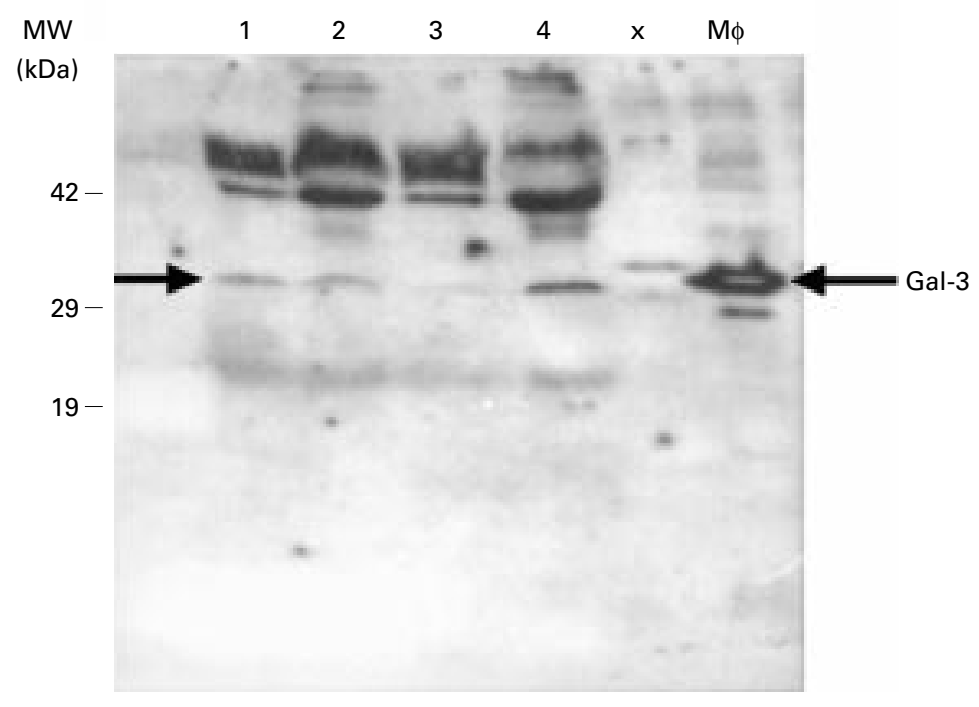

Figure 1 Western blotting for detection of galectin-3 using polyclonal antibody. MW: molecular weight (demonstrated according to position in acrylamide gel, because it is not visible in western analysis). Lanes 1, 2: tear fluid samples from patients with sarcoidosis, lane 3: tear fluid sample from patient with corneal degeneration, lane 4: tear sample from patient with adenovirus conjunctivitis, lane $M$ : extract from cultured human macrophage cell line producing galectin-3 used as a positive control. Only the tear samples from a few of the investigated patients are shown.

tear film, which has a protective, lubricative, and nutritive function. Both the corneal and conjunctival epithelia form the biodefence system of the anterior surface of the eye. The epithelium, together with the tears, has a pivotal role in maintaining the corneal integrity and its constituents affect eye surface immunology and responses to inflammation. Since galectin-3 is expressed by the human corneal epithelium and binds lipopolysaccharides purified from Pseudomonas aeruginosa as was demonstrated by the multiple inhibition assay, ${ }^{14}$ the participation of galectin-3 in eye surface biology is likely and its role as a member of the multiple adhesion family can be expected. The glycoconjugates represent the important component of the cell surface and no data about galectin-3 reactive glycoligands on the ocular surface epithelia are available. This knowledge is important for a rational explanation of the role of this endogenous lectin in eye physiology and pathology. Thus, we investigated the occurrence of this protein in the cornea, conjunctiva, and tears using a specific antibody. Moreover, we employed labelled galectin to analyse the binding sites for an endogenous lectin in this system. The employment of galectin-3 as a probe represents an important step to infer the presence of potential binding sites for this molecule, which can be important in elucidating its biological function in the eye.

\section{Materials and methods}

MATERIALS

Samples of normal corneas $(n=3)$, conjunctivas $(\mathrm{n}=4)$, and lacrimal gland $(\mathrm{n}=1)$ were obtained post mortem from donors without eye problems. The conjunctiva of a patient suffering with Stevens-Johnson syndrome $(n=1)$ was taken by biopsy. All samples were obtained after receiving the consent forms from the donors. The tear fluid samples (volume 5-12 $\mu \mathrm{l})$ were collected from normal, healthy people ( $\mathrm{n}=4$ ) without applying an irritant. The same volume of pathological tears (bullous keratopathy, $\mathrm{n}=1$, ocular manifestation of sarcoidosis, $\mathrm{n}=3$, chronic blepharitis, $\mathrm{n}=1$, toxic conjunctivitis $n=1$, adenoviral conjunctivitis, $\mathrm{n}=1$, pellucid marginal corneal degeneration, $\mathrm{n}=1$, alkali burn of cornea treated with corticosteroids, $\mathrm{n}=1$ ) was collected from patients as described. ${ }^{17}$

WESTERN BLOT ANALYSIS OF GALECTIN-3

PRESENT IN TEAR FLUID

The cells (if any) were separated from tear fluid by low rate centrifugation (minicentrifuge Qualitron, Sigma, Prague, Czech Republic) preventing cell damage and contamination of samples by galectin- 3 content of these cells. The samples were then stored at $-20^{\circ} \mathrm{C}$. Upon analysis, samples were combined with a sample buffer, denatured for 3 minutes at $100^{\circ} \mathrm{C}$, and centrifuged for 10 minutes at $16000 \mathrm{~g}$. Supernatants $(12 \mu \mathrm{l}$ of each samples) were then resolved by $14 \%$ SDS-PAGE. ${ }^{18}$ After electrophoresis, the resolved proteins were transferred to a nitrocellulose membrane, and a western blot analysis was performed as previously described..$^{19}$ Galectin-3 was detected with a rabbit polyclonal antibody ${ }^{7}$ (dilution 1:500) and peroxidase conjugated goat anti-rabbit IgG (Cappel Research Products, USA; dilution 1:5000) using enhanced chemiluminescence (ECL, Amersham, Pharmacia, Bioteck, Freiburg, Germany). Extract of cells from mouse macrophage line J774.GB producing galectin-3 was used as a positive control. In addition, a monoclonal mouse anti-galectin-3 antibody, A1D6 ${ }^{20}$ (dilution 1:150), a secondary antibody SwAM-Px (Temda, Prague, Czech Republic; dilution 1:200 000), and enhanced chemiluminescence (SuperSignal Wets Femto, Pierce) were used in a separate western blot.

IMMUNOFLUORESCENCE ANALYSIS AND LECTIN HISTOCHEMISTRY

Tissue section analysis

Specimens for histochemical investigation were exposed to Tissue Tek (Sakura-Finetek Europe BV Zoeterwoude, Netherlands) as a cryoprotective agent for 2 hours at $4^{\circ} \mathrm{C}$, then frozen in liquid nitrogen and stored in $-70{ }^{\circ} \mathrm{C}$. Cryostat sections (10 $\mu \mathrm{m}$; Cryocut-E, Reichert, Vienna, Austria) were fixed with $2 \%$ paraformaldehyde in PBS ( $\mathrm{pH} 7.2$ ) for 5 minutes; $0.1 \%$ bovine serum albumin in PBS was used as a blocking agent. After the extensive washing in TBS $(\mathrm{pH}$ 7.4), the specimens were stained for detection of galectin-3 with a monoclonal mouse antigalectin-3 antibody, A1D6. ${ }^{20}$ The desmosomal protein desmoglein was detected by using a commercial monoclonal antibody (Progen, Heidelberg, Germany). The cytokeratins in the lacrimal gland were detected by the monoclonal antibody LP-34 (Dako, Glostrup, Denmark) recognising a wide panel of cytokeratin types. FITC conjugated swine anti-mouse antibody (SwAM-FITC, Temda, Prague, Czech Republic) or swine anti-rabbit antibody 


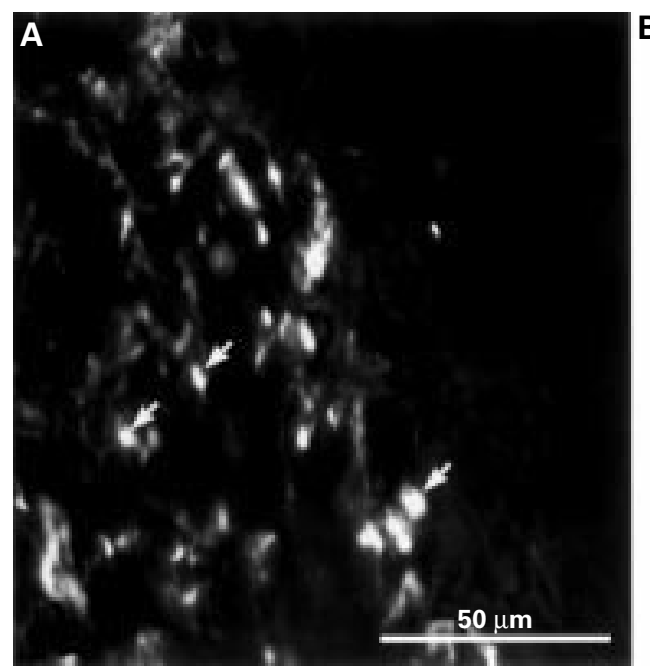

Figure 2 Positivity of inflammatory and goblet cells for galectin-3 detected by A1D6 antibody. Inflammatory cells in conjunctival stroma from a patient with Stevens-fohnson syndrome (A) expressed galectin-3 similarly to leucocytes (B) harvested from another patient with galectin-3 in tear fluid. The inflammatory cells infiltrating the cornea express the galectin-3 in cytoplasm in contrast with absence of galectin-3 in conjunctival epithelium. Immunofluorescence and immunoperoxidase detection of galectin-3, scale $50 \mu \mathrm{m}$ (A) and $10 \mu \mathrm{m}(B)$.
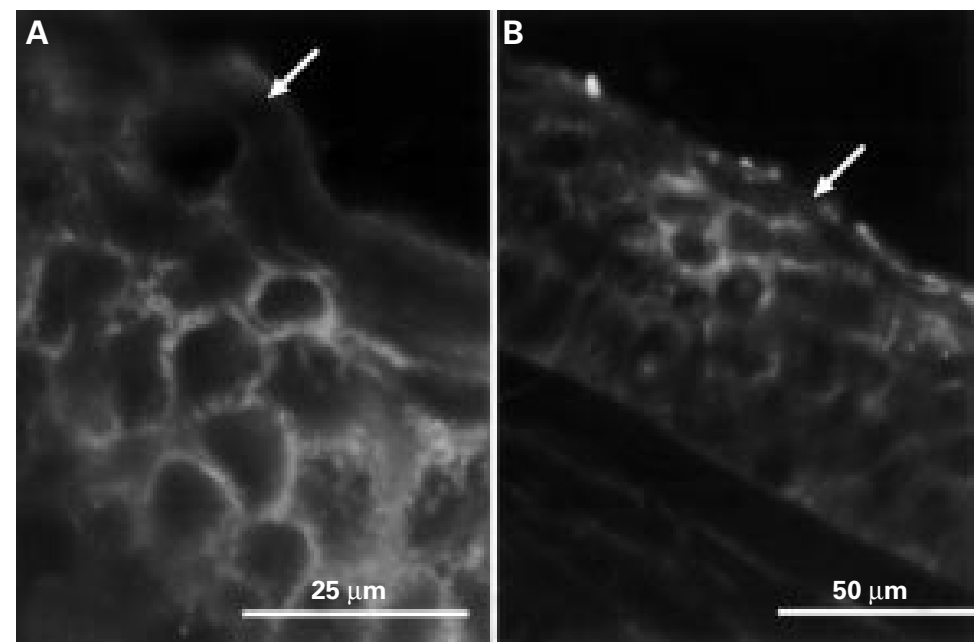

Figure 3 Galectin-3 expression in conjunctiva and cornea. Conjunctival $(A)$ and corneal (B) areas are positive in the immunohistochemical analysis. The galectin-3 was expressed predominantly on the cell surface. However, the expression of galectin-3 in the superficial part of conjunctiva was non-uniform; some areas were galectin-3 negative. The surface of corneal and conjunctival epithelium is marked by arrows. Detection of galectin-3 using A1D6 antibody, scale $25 \mu \mathrm{m}(A)$ and $50 \mu m(B)$.

(SwAR-FITC, Temda, Prague, Czech Republic) diluted 1:10 was employed as the second step reagent. If the peroxidase labelled second step antibody was used, the Sigma Fast system (Sigma, Prague, Czech Republic) with the diaminobenzidine tetrahydrochloride as substrate was employed to visualise the localisation of the bound antibody.

Galectin-3 binding sites were visualised by the lectin histochemical procedure using the biotinylated recombinant lectin as a probe. ${ }^{21-24}$ The ExtrAvidin-TRITC was employed as a second step reagent. The double labelling technology at one cell level ${ }^{24}$ was used to visualise desmoglein and galectin-3 binding sites simultaneously in one specimen.

The omission of the first step antibody or preincubation of galectin-3 with lactose as a competitive inhibitor to block carbohydrate dependent binding was used in control experiments to test the specificity of the immunohistochemical and lectin histochemical procedures. The specimens were mounted by Vectashield (Vector Laboratories, Burlingame, CA, USA). An Optiphot-2 (Nikon) fluorescence microscope and computer assisted image analysis system equipped with a CCD camera (Cohu) (Lucia, Laboratory Imaging, Prague, Czech Republic) was used for detection of signals.

\section{Tear sample cytology}

The teardrops containing cells (received as described above) were smeared on the surface of supporting glass and processed as described above for the detection of galectin-3. The cell types present in tear fluid were evaluated according to characteristic morphological features.

\section{Results}

The known secretion of galectins by other cells (macrophages, polymorphonuclear leucocytes, and epithelia) makes it likely that galectin-3 is present in tear fluid. Indeed, tears harvested from the eyes of patients with ocular inflammation contained galectin-3, although tears from healthy volunteers, the patient with corneal degeneration and alkali burned cornea (antibiotic and steroid treatment) did not (Fig 1). As detected by a rabbit polyclonal (or mouse monoclonal) antibody against gal-3, a band of apparent molecular weight around $30 \mathrm{kDa}$ was found in pathological tears samples as well as in control macrophages. Additional bands of higher molecular weight are at the position of immunoglobulins that are recognised by the secondary antibody that was not pre-adsorbed with human immunoglobulins. In a control western blot with mouse monoclonal antibody against gal-3 and another secondary antibody, similar bands were found (not shown).

Inflammatory cells (granulocytes and macrophages) that express galectin-3 were found in tear fluid samples which were positive for galectin-3 (Fig 2).

The immunofluorescence analysis with monoclonal and polyclonal antibodies against galectin-3 allowed the detection of galectin-3 in corneal and conjunctival epithelium. This protein was mainly present on the cell surface (Fig 3). The results demonstrated no differences if the monoclonal and polyclonal antibodies were used. No expression of galectin-3 was observed in the cells of the lacrimal gland, although cytokeratin expression monitored by the monoclonal antibody LP34 was clearly visible, indicating that the glandular proteins were not autolysed post mortem (not shown).

To show whether the cells also express binding sites for this lectin, it is necessary to analyse the cells with the labelled galectin-3. Biotinylated galectin- 3 was found to bind to the cell surface in normal corneal and conjunctival epithelium. The distribution of galectin-3 binding sites on corneal epithelium was uniform, whereas that on conjunctiva was rather irregular. The conjunctival epithelium from the patient with Stevens-Johnson syndrome expressed no galectin- 3 reactive binding 


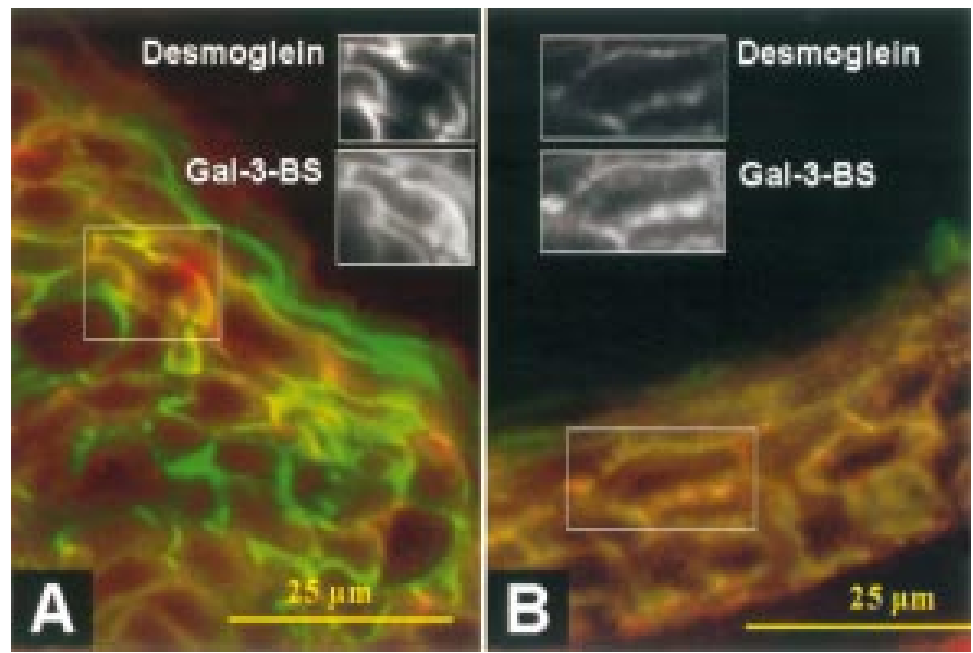

Figure 4 Co-localisation of galectin-3 binding sites with desmoglein in conjunctiva $(A)$ and cornea (B). The binding sites for galectin-3 (red signal) co-localised with expression of the desmosomal protein desmoglein (green signal). Desmoglein was detected by

immunohistochemistry and galectin-3 reactive glycloligands by lectin histochemistry, scale $25 \mu \mathrm{m}(A, B)$.

sites (not shown) although the macrophages under the epithelium were intensely stained by antibody against galectin-3 (Fig 2A) and expressed the galectin- 3 reactive binding sites. A remarkably strict co-localisation of galectin-3 reactive glycoligands with desmoglein was found in corneal and conjunctival epithelia (Fig 4). The employment of lactose as a competitive inhibitor completely blocked the binding of biotinylated galectin-3 to studied epithelia of the cornea and conjunctiva.

\section{Discussion}

Although galectin-3 is known to be secreted by various cells in vitro, in contrast with patients with ocular surface inflammation, no galectin-3 was found in tears harvested from healthy volunteers. Expression of galectin-3 was found in corneal and conjunctival epithelium in the normal eye and in leucocytes isolated from tears harvested from the galectin-3 positive eyes suffering from inflammation. Inflammatory cells infiltrating the conjunctival stroma from the patient with StevensJohnson were also highly positive for galectin-3. The lacrimal gland expressed no galectin-3 and therefore this gland is not a likely source of this lectin in the tear film. The non-pathological corneal as well as conjunctival epithelium expresses galectin-3, but the tears harvested from healthy volunteers contain no galectin-3. To infer presence of binding sites for the endogenous lectins, we prepared a biotinylated protein instead of a galactoside binding plant agglutinin, because carbohydrate fine specificities of two lectins can differ. ${ }^{25}$ Hypothetically, galectin-3 binding sites expressed in both the corneal and conjunctival epithelium appear positioned to immobilise galectin- 3 produced and exported from the cell to the cell surface. This phenomenon might explain the absence of galectin-3 in the tear film of healthy people, and could be of general relevance, because squamous epithelia, such as epidermis and oral mucosa, express galectin- 3 and galectin- 3 reactive glycoligands in a similar pattern. $^{21}{ }^{24}$ The co-localisation of galectin-3 reactive glycoligands with desmoglein suggests a participation of this endogenous lectin in intercellular contacts of the desmosomal type in the studied epithelia. However, this result must be verified. The irregular pattern of the accessible galectin-3 reactive glycoligands in conjunctiva can reflect the non-uniform distribution of these ligands in the conjunctiva or partial inaccessibility as a result of occupancy of some ligands by the endogenous lectin. This observation together with the ability of galectin-3 produced by corneal epithelial cells to immobilise bacterial lipopolysaccharides ${ }^{14}$ suggest a role for galectin-3 in the control mechanisms of the eye surface integrity and protection. In addition to epithelial cells, inflammatory cells such as polymorphonuclear leucocytes and macrophages are also known as producers of galectin-3. ${ }^{26}{ }^{27}$ These cells may be a source of galectin-3 in the tear film under pathological conditions.

In conclusion, this study shows the presence of galectin- 3 in the tear film in pathological eyes and reveals a difference from the normal condition. This, together with the initial monitoring of the lectin and binding site by immunohistochemistry and lectin histochemistry respectively, should prompt the elucidation of the functional role of the galectin at this location.

This study was supported by the grant agency of the Czech Republic project No 203/00/1310, the Academy of Sciences of the Czech Republic project No S40500005, and the Ministry of Public Health of the Czech Republic No ND 6340-3/2000. The authors are grateful to Eva Vancová for her excellent technical assistance.

1 Gabius H-J. Biological information transfer beyond the genetic code:the sugar code. Naturwissenschaften $2000 ; 87: 108-21$

2 Gabius H-J. Animal lectins. Eur f Biochem 1997;243:54376.

3 Panjwani N, Baum J. Lectin receptors of normal and dystrophic cornea. Acta Opthalmology (Suppl) 1989;192: 171-3.

4 Bishop PN, Bonshek RE, Jones CJ, et al. Lectin binding sites in normal, scarred and lattice dystrophy corneas. Br f Ophthalmol 1991;75:22-7.

5 Lange W, Debbage PL, Basting C, et al. Neoglycoprotein binding distinguish distinct zones in the epithelia of the eye. I Anat $1989 ; 166: 243-52$.

6 Hirabayashi J, ed. Recent topics on galectins. Trends Glycosci Glycotechnol 1997;9:1-180.

7 Kaltner H, Stierstorfer B. Animal lectins as cell adhesion molecules. Acta Anat 1998;161:162-79.

8 André S, Kojima S, Yamazaki N, et al. Galectins-1 and -3 and their ligands in tumor biology. $\mathcal{F}$ Cancer Res Clin Oncol 1999;125:461-74.

9 Adams L, Keneth Scoth G, Weiberg C. Biphasic modulation of cell growth recombinant human galectin-1. Biophys Acta 1996;1312:137-44.

10 Inohara H, Akahani S, Raz A. Galectin-3 stimulates cell proliferation. Exp Cell Res 1998;245:294-302.

11 Goldston SD, Lavin MF. Isolation of cDNA clone, encoding a human $\beta$ galactoside-binding protein overexpressed during glycocorticoid-induced cell death. Biochem Biophysic Res Commun 1991;178:746-50.

12 Sato S, Hughes RC. Regulation of secretion and surface expression of Mac- 2 and galactose-binding protein of macrophages. F Biol Chem 1994;269:4424-30.

13 Hsu DK, Yang R-Y, Pan Z, et al. Targeted disruption of the galectin-3 gene results in attenuated peritoneal inflammatory responses. Am f Pathol 2000;156:1073-83.

14 Gupta SK, Masinick S, Garrett M, et al. Pseudomonas aeruginosa lipopolisacharides bind galectin-3 and other human corneal epithelial proteins. Infect Immun 1997;65: 2747-53.

15 Smetana K Jr, Holíková Z, Klubal R, et al. Coexpression of binding sites for $\mathrm{A}(\mathrm{B})$ histo-blood group trisaccharide with galectin-3 and Lag antigen in human Langerhans cells. $\mathcal{f}$ Leukocyte Biol 1999;66:644-9.

16 Yang R-Y, Hsu KK, Liu F-T, et al. Expression of galectin-3 modulates T-cell growth and apoptosis. Proc Natl Acad Sci USA 1996:93:7737-42.

17 Bjerrum KB. Tear fluid analysis in patient with primary Sjögren's syndrome using lectin probes. Acta Ophthalmol Scand 1999;77:1-8. 
18 Laemmli UK. Cleavage of structural proteins during the assembly of the head of bacteriophage T4. Nature 1970;680-85.

19 Harlow E, Lane D. Immunobloting. In: Antibodies: a laboratory manual. Cold Spring Harbor: CSH Laboratory, $471-510$

20 Liu F-T, Hsu DK, Zuberi RI, et al. Modulation of functional properties of galectin-3 by monoclonal antibodies binding the non-lectin domains. Biochemistry 1996;35:6073-9.

21 Holíková Z, Smetana K Jr, Burchert M, et al. Expression of gaslectin-3, galectin-3 binding epitopes and Gal/GalNAcbinding sites in keratinocytes of the adult human epidermis. El f Pathol Histol 1999;5.2:992-1003.

22 Gabius H-J, Gabius S. Chemical and biochemical strategies for the preparation of glycohistochemical probes and their application in lectinology. Adv Lectin Res 1992:5:123-57.

3 Schwarz G, fingerprinting in tumor diagnosis. Differential expression of galectin-3 andgalectin-3-binding sites, but not galectin-1, in benign vs. malignant uterine smooth muscle

24 Plzák J, Smetana K Jr, Betka J, et al. Endogenous lectins (galectins-1 and-3) as probes to detect differentiationdependent alterations in human squamous cell carcinomas of the oropharynx and larynx. Int F Mol Med 2000;5:369-72.

25 Solís D, Jimenéz-Barbero J, Kaltner H, et al. Towards defining the role of glycans as hardware in information storage and transfer: basic principles, Experimental approaches and recent progress. Cells Tissues Organs 2001;168:5-23.

26 Truong M-J, Gruart V, Kusnierz J-P, et al. Human neutrophils express immunoglobulin $\mathrm{E}$ (IgE)-binding protein (Mac-2/eBP) of the S-type lectin family: role in IgE-dependent activation. $\mathcal{F}$ Exp Med 1993;173:177-9.

27 Liu FT, Hsu DK, Zuberi RI, et al Expression and function of galectin-3, a $\beta$-galactoside-binding lectin, in human monocytes and macrophages. Am f Pathol 1995;147:1016-28.

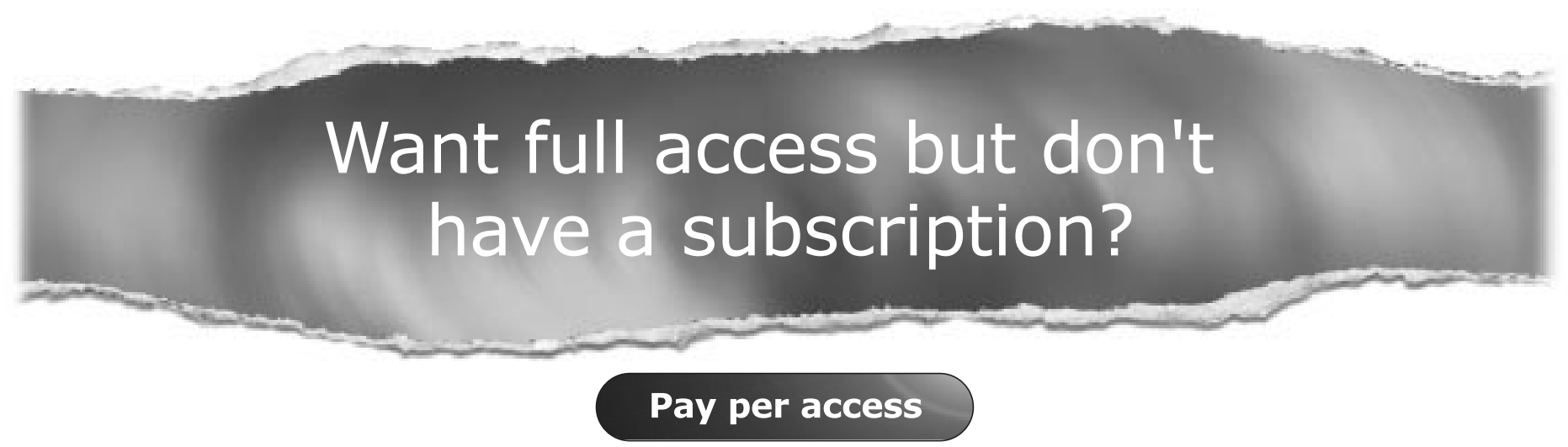

For just US $\$ 25$ you can have instant access to the whole website for 30 days. During this time you will be able to access the full text for all issues (including supplements) available. You will also be able to download and print any relevant pdf files for personal use, and take advantage of all the special features British Journal of Ophthalmology online has to offer.

\section{www.bjophthalmol.com}

